\title{
A new Vaejovis C.L. Koch, I 836, the second known vorhiesi group species from the Santa Catalina Mountains of Arizona (Scorpiones, Vaejovidae)
}

\author{
Richard F. Ayrey ${ }^{1, \dagger}$, Michael M. Webber ${ }^{2, \ddagger}$ \\ I P.O. Box 2236, Flagstaff, Arizona 86003, USA 2 School of Life Sciences, University of Nevada Las Vegas, \\ 4505 South Maryland Parkway, Las Vegas, Nevada 89154-4004, USA \\ † urn:lsid:zoobank.org:author:77A341F8-F8DA-41A8-BFB9-48D502F0E006 \\ † urn:lsid:zoobank.org:author:F4155CC7-5BB4-455B-9373-AB179B4D1FC4 \\ Corresponding author: Richard F. Ayrey (flagrich@azscorpion.com)
}

Academic editor: W. Lourenço| Received 13 December 2012 | Accepted 23 January 2013 | Published 18 February 2013

urn:lsid:zoobank.org:pub:FE68D993-194C-4A8C-BF38-DE8ECB6FCC7F

Citation: Ayrey RF, Webber MM (2013) A new Vaejovis C.L. Koch, 1836, the second known vorhiesi group species from the Santa Catalina Mountains of Arizona (Scorpiones, Vaejovidae). ZooKeys 270: 21-35. doi: 10.3897/zookeys.270.4500

\begin{abstract}
A new species of the vorbiesi group of Vaejovis C.L. Koch, 1836, Vaejovis brysoni sp. n., is described from the Santa Catalina Mountains in southern Arizona. Vaejovis deboerae Ayrey also inhabits this mountain range, making this the first documented case of two vorhiesi group species distributed on the same mountain. When compared to all other vorbiesi group species, Vaejovis brysoni sp. $\mathbf{n}$. is distinct based on several combinations of morphological characters and morphometric ratios.
\end{abstract}

\section{Keywords}

Madrean sky islands, speciation, taxonomy, Vaejovis vorhiesi group 


\section{Introduction}

For over 50 years, only four species of montane scorpions in the specious genus Vaejovis were known from the topographically complex states of Arizona, New Mexico and Sonora. That number has more than doubled over the past six years, with a total of 13 species now known (see Graham et al. 2012), all belonging to the Vaejovis vorhiesi group (Soleglad and Fet 2008). All 13 species have allopatric distributions in Arizona (Sissom et al. 2012), and no records of co-occurrence have been documented. Interestingly, however, several species are distributed across overlapping ecological communities. For example, Vaejovis jonesi Stahnke (1940) inhabits rocky juniper woodlands on the Colorado Plateau, and Vaejovis lapidicola Stahnke (1940) is distributed across pine-oak woodlands along the southern edge of the Colorado Plateau. These ecological communities overlap across the rim of the Mogollon Plateau, yet to date $V$. jonesi and V. lapidicola have not been found syntopically. Vaejovis deboerae Ayrey (2009) was recently described from the high pine-oak forests of the Santa Catalina Mountains in southern Arizona. The type series was collected at an elevation of $2142 \mathrm{~m}$. Other records suggest that $V$. deboerae may range as high as $2800 \mathrm{~m}$ and as low as $1520 \mathrm{~m}$ (Sissom et al. 2012). This vertical distribution encompasses a gradient of ecological communities, ranging from cold pine forest on the high peaks of the Santa Catalina to drier juniper desert scrub in the lower canyons. Recent collecting in the Santa Catalina along the transition zone between desert grassland and pine-oak forest revealed a distinct second species of $V$. vorhiesi group scorpion. Here we describe this new species, which represents the first record of two vorhiesi group species inhabiting the same mountain range.

\section{Materials and methods}

The systematics adhered to in this paper follow the classification established in Fet and Soleglad (2005) and as modified by Soleglad and Fet (2006), Graham and Soleglad (2007), Fet and Soleglad (2007), Soleglad et al. (2007), and Soleglad and Fet (2008). Measurements are as described in Stahnke (1970), trichobothrial patterns are as in Vachon (1974), and pedipalp finger dentition follows Soleglad and Sissom (2001).

Acronyms of depositories - RFA, Richard F. Ayrey; MES, Michael E. Soleglad; USNM, United States National Museum, Smithsonian.

Material - In addition to the type material listed below for the new species, the following additional specimens were examined:

Vaejovis brysoni sp. n. USA: Arizona: Pima Co.: above Molino Basin on Catalina Highway near Seven Cataracts Vista, Santa Catalina Mountains. $32.35796^{\circ} \mathrm{N}$ $110.72538^{\circ} \mathrm{W}, 1626$ m. 16 March 2012. R.W. Bryson Jr. 1 đ̊, 7 ㅇ (RFA). Same 
locality. 5 April 2012. R.W. Bryson Jr. \& D. Hartman 8 ㅇ (RFA). Same locality.

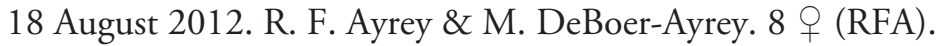

Vaejovis cashi Graham, 2007. USA: Arizona: Cochise Co.: Cave Creek Canyon, Chiricahua Mountains. 2 August 2008. R. F. Ayrey \& M. M. DeBoer-Ayrey 4 $\widehat{\jmath}, 4$ 우 (RFA). Same locality. 23 August 2011. R. F. Ayrey \& M. M. DeBoer-Ayrey 3 đ, 4 + (RFA).

Vaejovis crumpi Ayrey et Soleglad, 2011. USA: Arizona: Yavapai Co.: by Lynx Lake, Prescott. 14 August 2008. R. F. Ayrey \& M. M. DeBoer-Ayrey 30 , 5 q topotypes (RFA). Same locality. 14 September 2009. R. F. Ayrey \& M. M. DeBoer-Ayrey 4 J, 4 q (RFA). Same locality. 8 August 2010. R. F. Ayrey \& M. M. DeBoer-Ayrey 3 त, 5 우 (RFA).

Vaejovis deboerae Ayrey, 2009. USA: Arizona: Pima Co.: Rose Canyon Campground, Santa Catalina Mountains. 28 August 2011. R. F. Ayrey \& M. M. DeBoer-Ayrey

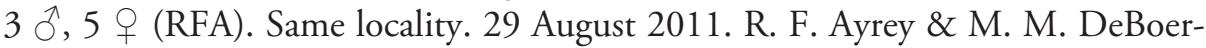
Ayrey $4 \hat{0}, 4$ ㅇ (RFA).

Vaejovis electrum Hughes, 2011. USA: Arizona: Graham Co.: Upper Arcadia Campground, Mount Graham. 17 July 2009. R. F. Ayrey \& M. M. DeBoer-Ayrey 2 O, 6 + (RFA). USA: Arizona: Graham Co.: 9415 feet asl, Mt Graham Hwy., Mt. Graham. 18 July 2009. R. F. Ayrey \& M. M. DeBoer-Ayrey 1 ○े, 4 q (RFA).

Vaejovis feti Graham, 2007. USA: New Mexico: Meadow Creek, Black Mountains. 6 July 1978. M. H. Muma 4 ô, 3 q (MES).

Vaejovis halli Ayrey, 2012. USA: Arizona: Gila Co.: Mount Ord. 11 September 2010. R. F. Ayrey \& M. M. DeBoer-Ayrey 2 O, 6 q, paratypes (RFA). Same locality. 2 May 2011. R. F. Ayrey \& M. M. DeBoer-Ayrey $3 \hat{\jmath}, 5$ ㅇ, paratypes (RFA).

Vaejovis jonesi Stahnke, 1940. USA: Arizona: Coconino County: near Wupatki National Monument. 1 April 2011. R. F. Ayrey. 1 topotype (RFA).

Vaejovis lapidicola Stahnke, 1940. USA: Arizona: Coconino County: Red Sandstone Quarry, Flagstaff. 1 June 2011. R. F. Ayrey \& M. M. DeBoer-Ayrey 1 đ,, 7 o topotypes (RFA).

Vaejovis paysonensis Soleglad, 1973. USA: Arizona: Coconino County: Control Road, 25 miles East of Payson. 5 July 2011. R. F. Ayrey \& M. M. DeBoer-Ayrey 1 ठૈ, 7 topotypes (RFA). Same locality. 6 July 2011. R. F. Ayrey \& M. M. DeBoerAyrey $2 \lambda, 6 q$ topotypes (RFA).

Vaejovis tenuipalpus Sissom et al., 2012. USA: Arizona: Mojave Co.: Getz Peak, Hualapai Mountains. 9 August 2009. R. F. Ayrey \& M. M. DeBoer-Ayrey 1 §,, 7 q paratopotypes (RFA).

Vaejovis vorhiesi Stahnke, 1940. USA: Arizona: Cochise Co.: Miller Canyon, Huachuca Mountains. 24 May 2011. R. F. Ayrey \& M. M. DeBoer-Ayrey 1 Oૈ, 7 q topotypes (RFA). Garden Canyon, Huachuca Mountains. 26 August 2011. R. F. Ayrey \& M. M. DeBoer-Ayrey 4 đ , 6 ㅇ (RFA). Lutz Canyon, Huachuca Mountains. 27 March 2011. R. F. Ayrey \& M. M. DeBoer-Ayrey 2 đ, 2 q (RFA). 


\section{Taxonomy}

Order Scorpiones C. L. Koch, 1850

Suborder Neoscorpiones Thorell et Lindström, 1885

Infraorder Orthosterni Pocock, 1911

Parvorder Iurida Soleglad et Fet, 2003

Superfamily Chactoidea Pocock, 1893

Family Vaejovidae Thorell, 1876

Subfamily Vaejovinae Thorell, 1876

\section{Vaejovis brysoni sp. $\mathrm{n}$.}

urn:lsid:zoobank.org:act:80FC6074-1CD9-4DED-B155-2F7FE348495C http://species-id.net/wiki/Vaejovis_brysoni

Figs 1-10, 12; Table 1

Type material. Female holotype. USA: Arizona: Pima Co.: above Molino Basin on Catalina Highway near Seven Cataracts Vista, Santa Catalina Mountains. $32.35796^{\circ} \mathrm{N}$, $110.72538^{\circ} \mathrm{W}, 1626 \mathrm{~m} .16$ March 2012. R.W. Bryson Jr. (RFA specimen number 632, deposited in USNM). Paratypes. Same locality as holotype. 16 March 2012. R.W. Bryson Jr. 1 ô (RFA specimen number 633) 2 ㅇ (RFA specimen numbers 634 and 635). 17 August 2012. R. F. Ayrey. 1 क (RFA specimen number 643).

Etymology. The specific epithet is a patronym honoring our colleague Dr. Robert W. Bryson, Jr., the collector of the holotype.

Diagnosis. Relatively small-bodied scorpion from the Seven Cataracts Overlook area of the Santa Catalina Mountains, southern Arizona (total body length of the female holotype is $27.50 \mathrm{~mm}$ ). Color is light to medium brown, light brown to yellow on the legs, with underlying dark mottling on carapace and mesosoma. Metasoma is light brown with darker carinae.

Significant characters that distinguish $V$. brysoni sp. n. from other known species in the vorhiesi group are described below.

Vaejovis jonesi, V. lapidicola, V. paysonensis, V. crumpi, and V. bigelowi all possess 7 inner denticles $(I D)$ on the chela movable finger, not 6 as in $V$. brysoni sp. n.. The new species can be distinguished from $V$. halli by having significantly larger metasomal segment L/W ratios on I, II, and V (Table 1). Vaejovis brysoni can be distinguished from $V$. bandido by having larger metasomal segment I L/W ratios in addition to larger fixed finger $\mathrm{L} /$ chela $\mathrm{L}$ ratios. Vaejovis brysoni sp. n. can be distinguished from $V$. deboerae by having a smaller and less-developed subaculear tubercle. Vaejovis brysoni sp. n. also have shorter total body lengths and shorter carapace lengths. In addition, $V$. deboerae have larger telson vesicle L/W ratios. However, $V$. brysoni sp. n. have larger metasomal segment I L/W ratios and larger fixed finger L/chela L ratios. Vaejovis brysoni sp. n. also have fewer pectinal teeth than $V$. deboerae. Vaejovis brysoni sp. n. can be distinguished from $V$. vorhiesi by having larger metasomal segments L/W ratios on I, II, and III. However, V. vorhiesi have larger chela L/W ratios. Vaejovis brysoni sp. n. also have 


\begin{tabular}{|c|c|c|c|c|c|c|c|c|c|c|c|c|}
\hline है & 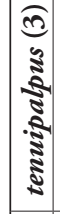 & 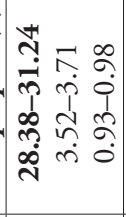 & $\begin{array}{l}\stackrel{+}{o} \\
\vdots \\
1 \\
\vdots \\
\vdots\end{array}$ & 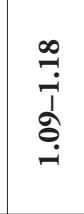 & กั. & \begin{tabular}{l}
$\infty$ \\
$\infty$ \\
\multirow{I}{+}{} \\
$\stackrel{1}{-}$
\end{tabular} & 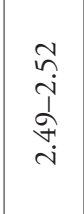 & 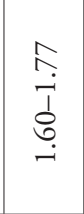 & $\mid \begin{array}{c}n \\
\infty \\
\dot{n} \\
1 \\
\infty \\
\infty \\
\dot{n}\end{array}$ & 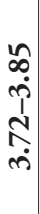 & 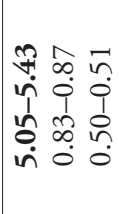 & 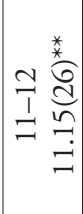 \\
\hline 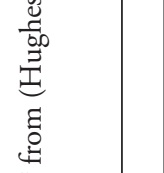 & $\mid$ & 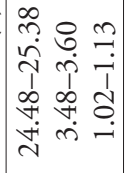 & $\begin{array}{l}\infty \\
\vdots \\
0 \\
1 \\
n \\
\vdots \\
0\end{array}$ & $\begin{array}{l}\infty \\
\infty \\
0 \\
1 \\
0 \\
0 \\
0\end{array}$ & 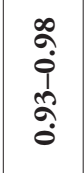 & 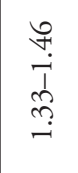 & 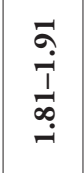 & 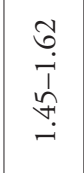 & $\begin{array}{l}2 \\
\dot{p} \\
\infty \\
\infty \\
\dot{v}\end{array}$ & $\begin{array}{l}\stackrel{+}{a} \\
\grave{i} \\
i \\
\infty \\
i \\
i\end{array}$ & 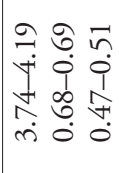 & 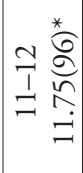 \\
\hline & कृ & 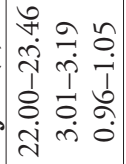 & $\begin{array}{l}\hat{n} \\
\hat{0} \\
1 \\
\vdots \\
0\end{array}$ & $\begin{array}{l}\hat{n} \\
\hat{0} \\
1 \\
\vdots \\
0\end{array}$ & $\begin{array}{l}n \\
\stackrel{1}{1} \\
1 \\
a \\
0\end{array}$ & $\begin{array}{l}8 \\
\stackrel{1}{-} \\
1 \\
n \\
n \\
-1\end{array}$ & 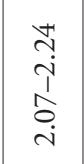 & 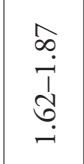 & $\begin{array}{c}F \\
\dot{p} \\
\dot{1} \\
\infty \\
\infty \\
i\end{array}$ & 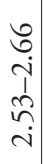 & 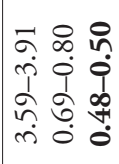 & $\begin{array}{l}0 \\
ㅇ \\
0\end{array}$ \\
\hline & 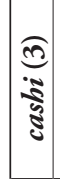 & 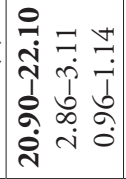 & $\begin{array}{l}0 \\
0 \\
0 \\
1 \\
\vdots \\
0\end{array}$ & \begin{tabular}{l}
$\stackrel{\infty}{0}$ \\
0 \\
\multirow{1}{1}{} \\
$\hat{0}$
\end{tabular} & $\begin{array}{l}\tilde{\sigma} \\
\hat{1} \\
o \\
0 \\
0\end{array}$ & $\begin{array}{l}\text { กे } \\
\stackrel{-}{\infty} \\
\stackrel{-}{-}\end{array}$ & $\begin{array}{l}n \\
\tilde{i} \\
i \\
\tilde{o} \\
i\end{array}$ & 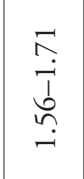 & 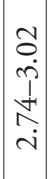 & $\begin{array}{l}m \\
0 \\
\dot{p} \\
b \\
\infty \\
\dot{v}\end{array}$ & 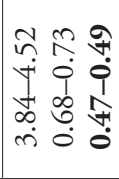 & 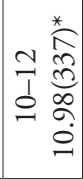 \\
\hline 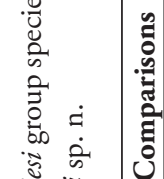 & 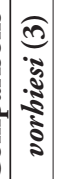 & 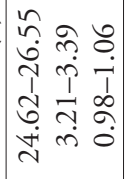 & $\begin{array}{l}\hat{n} \\
\hat{0} \\
\infty \\
0 \\
0 \\
0\end{array}$ & $\begin{array}{l}1 \\
\hat{0} \\
1 \\
n \\
\infty \\
0\end{array}$ & 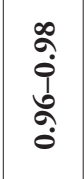 & $\begin{array}{l}\text { W़ } \\
\stackrel{-}{1} \\
\text { ले } \\
-\end{array}$ & 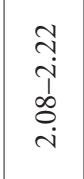 & 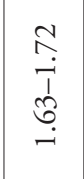 & $\begin{array}{c}\tilde{T} \\
\dot{p} \\
\hat{1} \\
\infty \\
i \\
\dot{\lambda}\end{array}$ & $\begin{array}{l}a \\
\dot{m} \\
\dot{\sim} \\
\stackrel{n}{m}\end{array}$ & 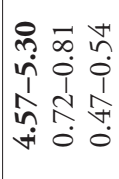 & 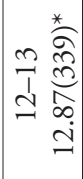 \\
\hline 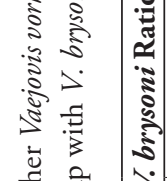 & $\begin{array}{c}0 \\
0 \\
\vdots \\
\vdots \\
\vdots \\
z \\
z \\
0\end{array}$ & 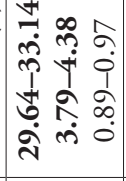 & $\begin{array}{l}\stackrel{\hat{T}}{\hat{0}} \\
\stackrel{1}{\hat{0}} \\
\hat{0}\end{array}$ & $\begin{array}{l}m \\
\stackrel{0}{1} \\
1 \\
\infty \\
0 \\
0\end{array}$ & $\begin{array}{l}\stackrel{+}{ \pm} \\
\stackrel{-}{I} \\
\stackrel{2}{0} \\
\stackrel{-}{-}\end{array}$ & 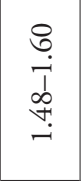 & $\begin{array}{l}\tilde{n} \\
i \\
i \\
\stackrel{i}{i}\end{array}$ & 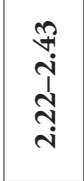 & $\mid \begin{array}{c}2 \\
\grave{i} \\
\dot{1} \\
\stackrel{+}{i} \\
i\end{array}$ & 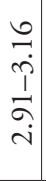 & 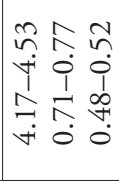 & 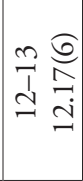 \\
\hline 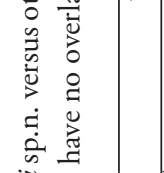 & 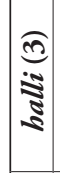 & 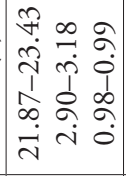 & $\begin{array}{l}\hat{N} \\
0 \\
1 \\
1 \\
0 \\
0\end{array}$ & $\begin{array}{l}\hat{\infty} \\
0 \\
1 \\
\hat{T} \\
\hat{0}\end{array}$ & \begin{tabular}{l}
8 \\
$\stackrel{1}{1}$ \\
\multirow{2}{0}{} \\
$\vdots$
\end{tabular} & 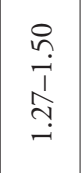 & 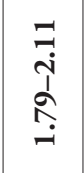 & 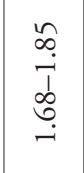 & $\mid \begin{array}{c}m \\
\tilde{n} \\
n \\
n \\
\infty \\
\infty \\
i\end{array}$ & $\begin{array}{l}\tilde{\sigma} \\
\dot{p} \\
\hat{\sigma} \\
\infty \\
\dot{\nu}\end{array}$ & 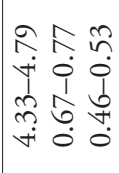 & 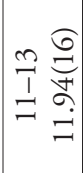 \\
\hline 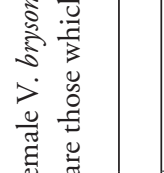 & 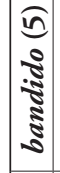 & 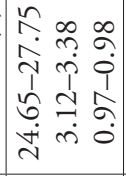 & 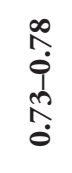 & 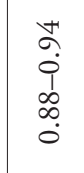 & $\begin{array}{l}\stackrel{t}{0} \\
\stackrel{1}{1} \\
\stackrel{2}{0} \\
0\end{array}$ & 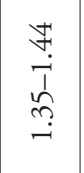 & 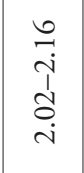 & 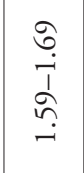 & $\mid \begin{array}{c}0 \\
0 \\
i \\
b \\
b \\
\dot{d}\end{array}$ & $\begin{array}{l}\vec{\sim} \\
\tilde{n} \\
\tilde{n} \\
\infty \\
\dot{v}\end{array}$ & 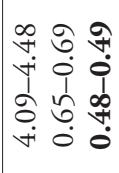 & $\begin{array}{l}\sim 0 \\
\\
\beth \\
= \\
=\end{array}$ \\
\hline 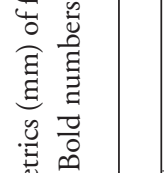 & 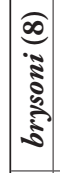 & 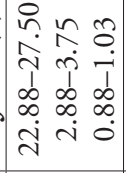 & $\begin{array}{l}8 \\
\stackrel{1}{1} \\
2 \\
\check{0}\end{array}$ & 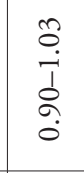 & 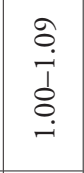 & 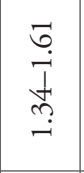 & $\begin{array}{l}\text { ô } \\
\stackrel{i}{1} \\
\stackrel{1}{n} \\
\stackrel{i}{i}\end{array}$ & 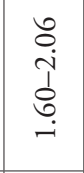 & $\left|\begin{array}{c}\tilde{z} \\
\dot{p} \\
1 \\
\infty \\
i \\
i\end{array}\right|$ & 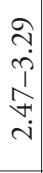 & 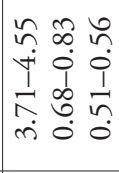 & 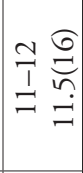 \\
\hline 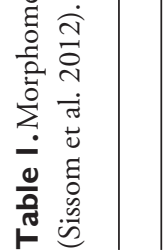 & 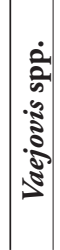 & 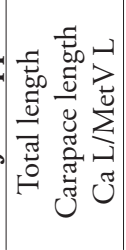 & 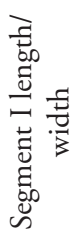 & 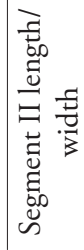 & 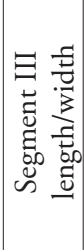 & 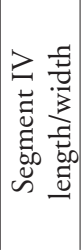 & 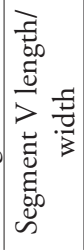 & 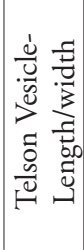 & 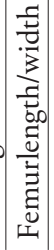 & 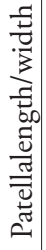 & 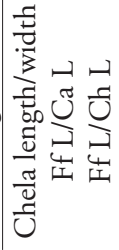 & 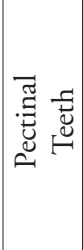 \\
\hline
\end{tabular}




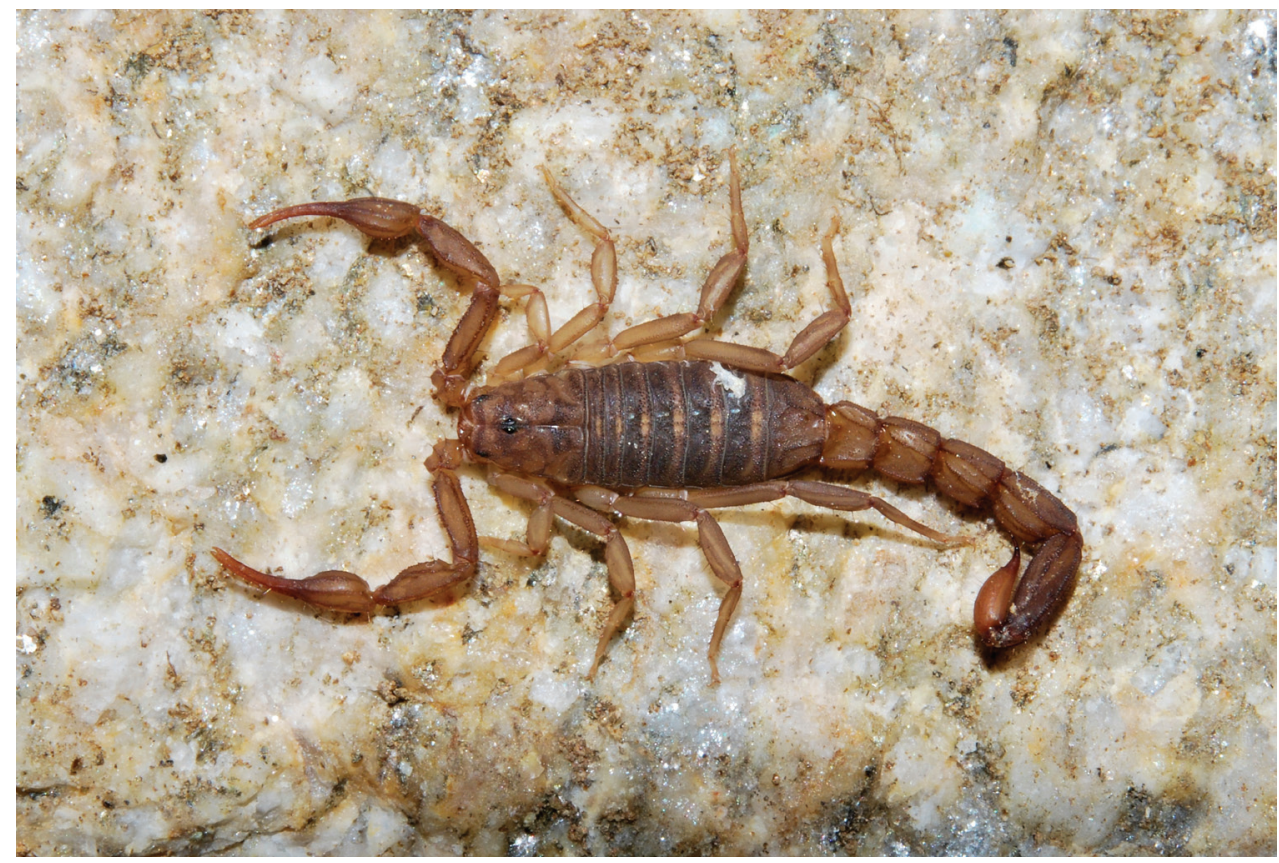

Figure I. Vaejovis brysoni sp. n., paratype female in natural habitat.

fewer pectinal teeth than $V$. vorhiesi. Vaejovis brysoni sp. n. can be distinguished from $V$. cash $i$ by having longer total body lengths, and larger metasomal segment L/W ratios on segments I, II, and III. In addition, V. brysoni sp. n. exhibit larger fixed finger L/ chela L ratios. Vaejovis brysoni sp. n. can be distinguished from $V$. feti by having larger metasomal segment I and II L/W ratios. Additionally, $V$. brysoni sp. n. also have larger fixed finger L/chela L ratios than $V$. feti. Vaejovis brysoni sp. n. can also be distinguished from $V$. feti by having a higher number of pectinal teeth. Vaejovis brysoni sp. n. can be distinguished from $V$. electrum by having larger metasomal segment L/W ratios on segments I, II, III, and V. In addition, V. brysoni sp. n. also have larger fixed finger L/chela L ratios than $V$. electrum. Finally, Vaejovis brysoni sp. n. can be distinguished from $V$. tenuipalpus by having smaller metasomal segment L/W ratios on segments II, III, and IV. Vaejovis tenuipalpus also have larger femur, patella, and chela L/W ratios.

Description of the holotype. Color of the holotype is light to medium brown, light brown to yellow on the legs, with underlying dark mottling on carapace and mesosoma. Metasoma is light brown with darker carinae. Metasomal segments are slightly wider than the vesicle. Small spinoid subaculear tubercle is present (Fig. 1). The pedipalp fixed finger has 5 to 6 ID denticles and movable finger has 6 ID denticles. Carapace: Anterior margin of the carapace is slightly emarginated, the posterior margin is straight. The carapace is moderately granular, with three lateral eyes present on each side. The median furrow is moderate and traverses the entire length of the carapace. The ratio of the location of the median eyes on the carapace (anterior edge/ 

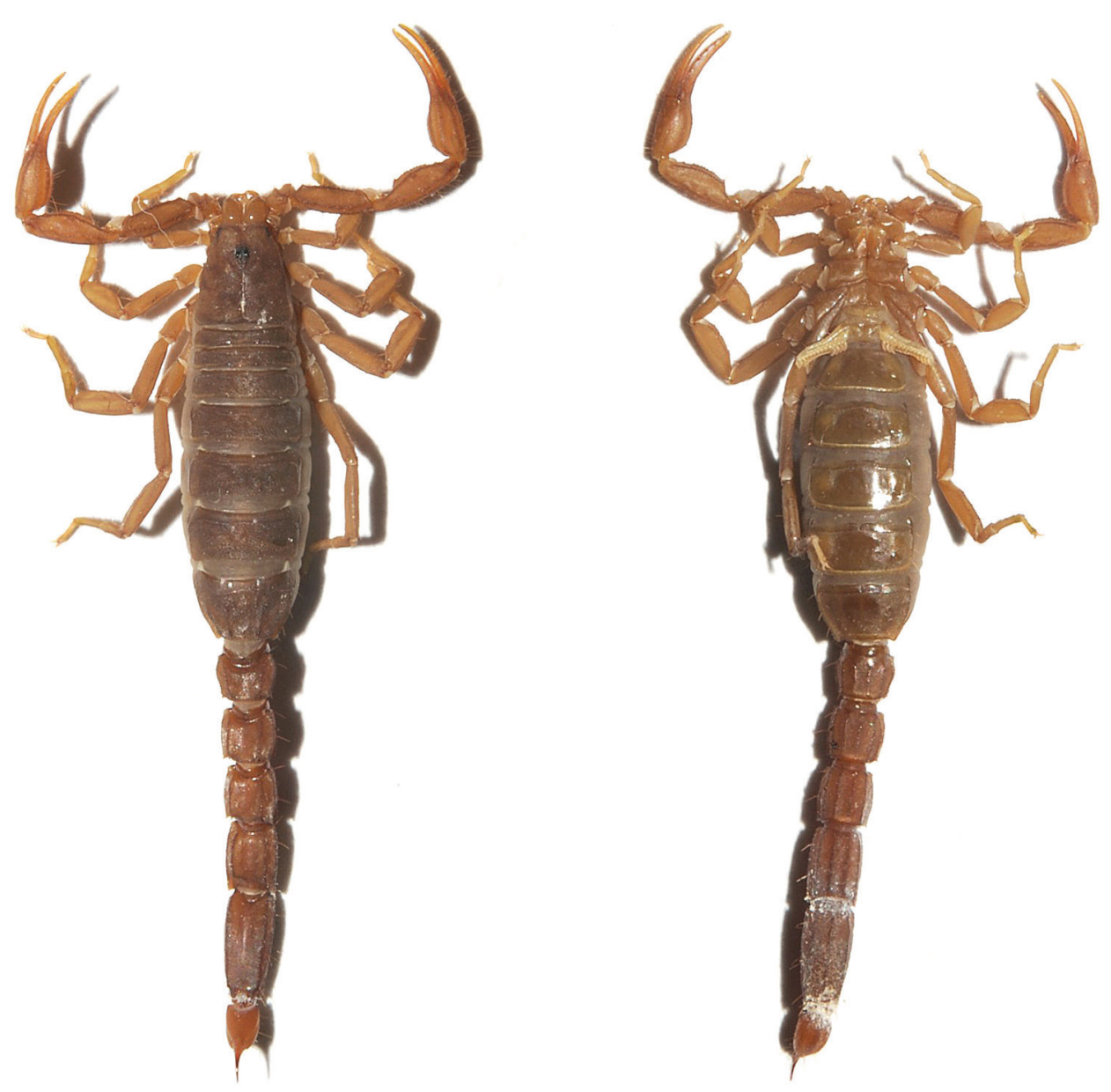

Figure 2. Vaejovis brysoni sp. n., paratype female dorsal and ventral views.

carapace length $0.73 / 3.75)=0.19$; carapace length/width at median eyes $3.75 / 2.35$ $=1.60$. The carapace is longer than metasomal segment $\mathrm{V}$. Mesosoma: Tergites are moderately granular with vestigial median carina on tergites I-VI. Tergite VII with weak median carina on anterior third and strong dorsal lateral and lateral supramedian granular carinae. Sternites I-V are finely granular and without carinae. Sternite $\mathrm{V}$ with weak granular ventral lateral carinae on middle $1 / 3$. Presternites are smooth. Spiracles are ovoid with median side rotated 35 degrees from posterior sternite margin. Sternites with variable number of microsetae. Pectines: Pectinal tooth count is11/12. All pectinal teeth have exterodistal angling with a large sensorial area. Middle lamellae are 6/6. Fulcra are present. Each fulcra with 1-3 central setae. Metasoma: The carapace of the holotype female is longer than the fifth metasomal segment. Ratio of segment I length/width 0.93; of segment II length/width 1.03; of segment III length/width 1.18; of segment IV length/width 1.50; of segment V length/width 2.32. Segments I-IV: 


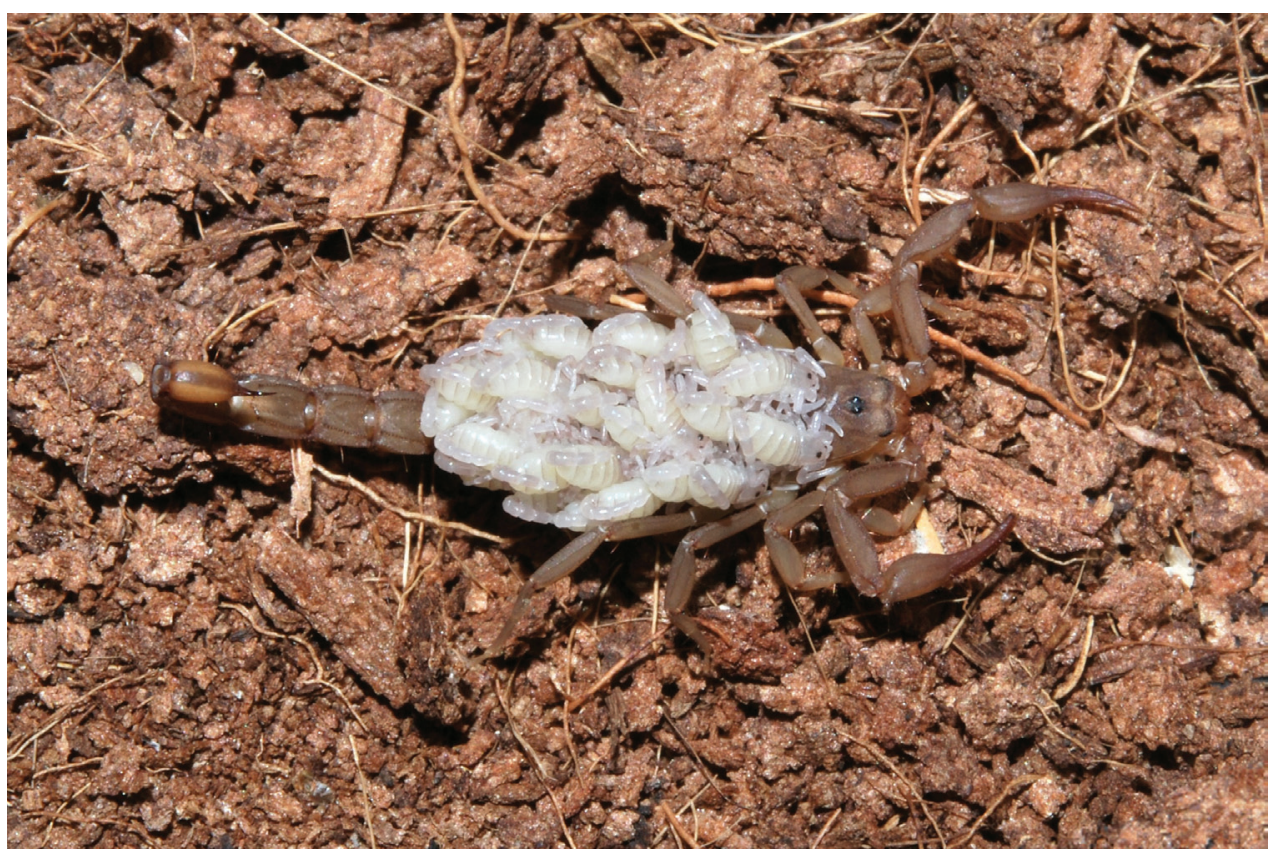

Figure 3. Vaejovis brysoni sp. n., paratype female with first instar juveniles.

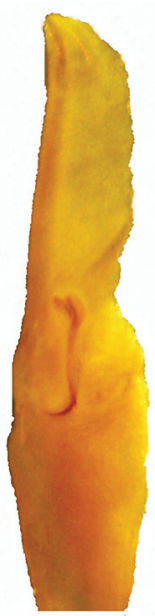

Figure 4. Vaejovis brysoni sp. n., paratype male right hemispermatophore.

dorsolateral carinae are strong and granular to slightly dentate, with the distal denticle of I-IV enlarged and spinoid. Lateral supramedian carinae I-IV are strong and crenulate, with enlarged spinoid distal denticle. Lateral inframedian carinae are moderately granular on posterior 4/5 of segment I, 4/5 of II, 1/2 of III, and nearly obsolete on segment IV. Ventrolateral carinae on segment I, II, and III are moderate and granular; on IV moderate, granular and slightly serrate. Ventral submedian carinae are weak on segment I, weak to moderate on II, moderate, granular to slightly serrate on III and 

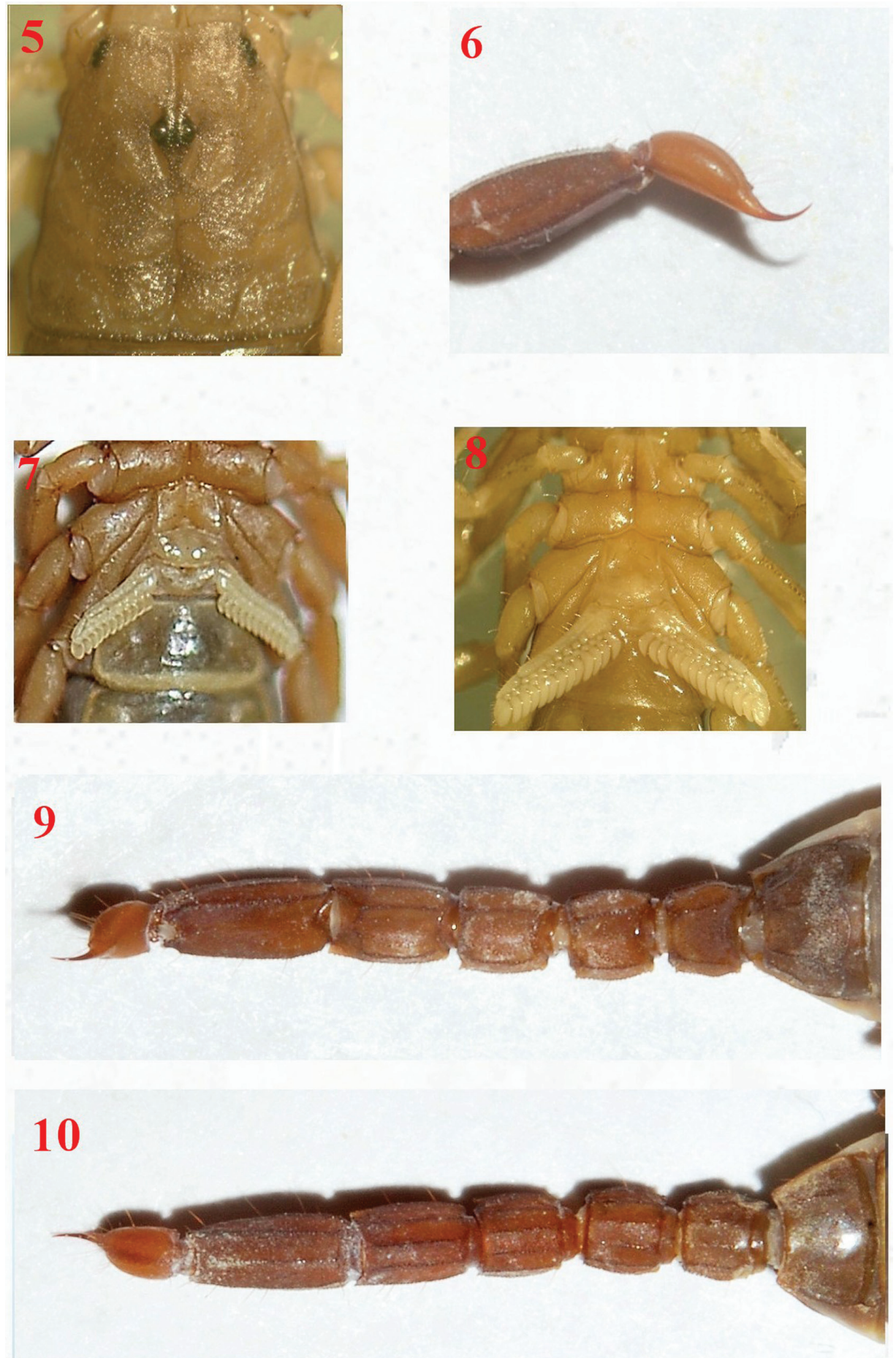

Figures 5-10. Vaejovis brysoni sp. n., paratype female carapace 5; telson 6; pectines 7; paratype male pectines and sternites 8; paratype female metasoma dorsal $\mathbf{9}$; and ventral $\mathbf{I 0}$. 
IV. The dorsal and lateral intercarinal spaces are very finely granular. Segment I-IV: ventral submedian setae count is $3 / 3$. Segment V: dorsolateral carinae are moderate and slightly serrate on anterior $1 / 3$. Lateromedian carinae are weak to moderate and granular on basal $3 / 5$, and obsolete on distal $2 / 5$. Ventrolateral and ventromedian carinae are strong and crenate to serrate. Intercarinal spaces are finely granular. Ventrolateral setae count 4/4. Telson: Smooth with four pairs of large setae on the ventral surface, three large setae are along both lateral edges of the vesicle with numerous smaller setae. A small spinoid subaculear tubercle is present. Chelicerae: The dorsal edge of movable cheliceral finger with two subdistal (sd) denticles. Ventral edge is smooth, with well developed serrula on distal half. Pedipalps: Trichobothrial pattern type C (Vachon 1974) (Fig. 12). Trichobothria $i b$ and it near base of fixed finger. Pedipalp ratios: chela length/width 4.00; femur length/width 2.69; patella length/width 2.59; fixed finger length/carapace length 0.68. Chela: Carinae are moderate. Fixed and movable finger median denticles (MD) are aligned and divided into 6 subrows by 5 outer denticles (OD) and usually 6 ID denticles. Femur: Dorsal internal and external are moderate and granular; ventral internal granular to crenulate; ventral external are slightly serrate; dorsal and ventral surfaces are covered with fine granules; external surface is smooth. Patella: Internal surface are covered with very strong dentate to serrate granules on the DPSc carina. Dorsal external and internal are moderate and granular. Ventral internal carinae are strong and granular. External surface is rounded with scattered granulation; dorsal and ventral surfaces are covered with minute granules. Legs: Ventral surface of tarsomere II with single median row of spinules terminating distally with one spinule pair.

Variability. Variability of fixed finger ID denticle count was found. For V. brysoni sp. n., fixed finger ID denticle counts ranged from $5(n=3)$ to $6(n=5)$. Variation also existed for female $V$. brysoni sp. $\mathrm{n}$. in the number of pectinal teeth $11 / 11(\mathrm{n}=2), 11 / 12$ $(\mathrm{n}=3), 12 / 11(\mathrm{n}=1), 12 / 12(\mathrm{n}=2)$ with a mean of 11.5 for females, and $13 / 14$ for the paratype male $(\mathrm{n}=1)$. In addition, there was variation in the number of middle lamellae $5 / 5(\mathrm{n}=1), 6 / 6(\mathrm{n}=5), 7 / 6(\mathrm{n}=1), 7 / 7(\mathrm{n}=1)$ and for the paratype male 8/9 $(\mathrm{n}=1)$. The right hemispermatophore was extracted from the paratype male. The right hemispermatophore is $3.10 \mathrm{~mm}$ in total length, and its lamina is $1.20 \mathrm{~mm}$ in length and 0.39 $\mathrm{mm}$ in width. The hemispermatophore is lightly sclerotized near the dorsal trough, and possesses a subtle distal crest on the inner distal aspect of the lamina. The lamellar hook is strong and widely bifurcated, and emanates from the dorsal trough. A medium, defined truncal flexure is visible on the external aspect of the trunk/lamina juncture. The male paratype also posseses an area of reduced pigmentation (white patch) on the posterior $1 / 4$ of the third sternal plate. (Graham and Bryson 2010).

Mensuration (mm). Female holotype: total length 27.5; carapace length 3.75; mesosoma length 8.13; metasoma length 15.63. Metasoma: segment I length/ width/depth 1.81/1.94/1.38; segment II length/width/depth 2.00/1.94/1.25; segment III length/width/depth 2.06/1.75/1.31; segment IV length/width/depth 2.63/1.75/1.25; segment $\mathrm{V}$ length/width/depth 3.63/1.56/1.25. Telson: length 3.50; vesicle length/width/depth 2.25/1.25/1.06; aculeus length 1.25. Pedipalps: 


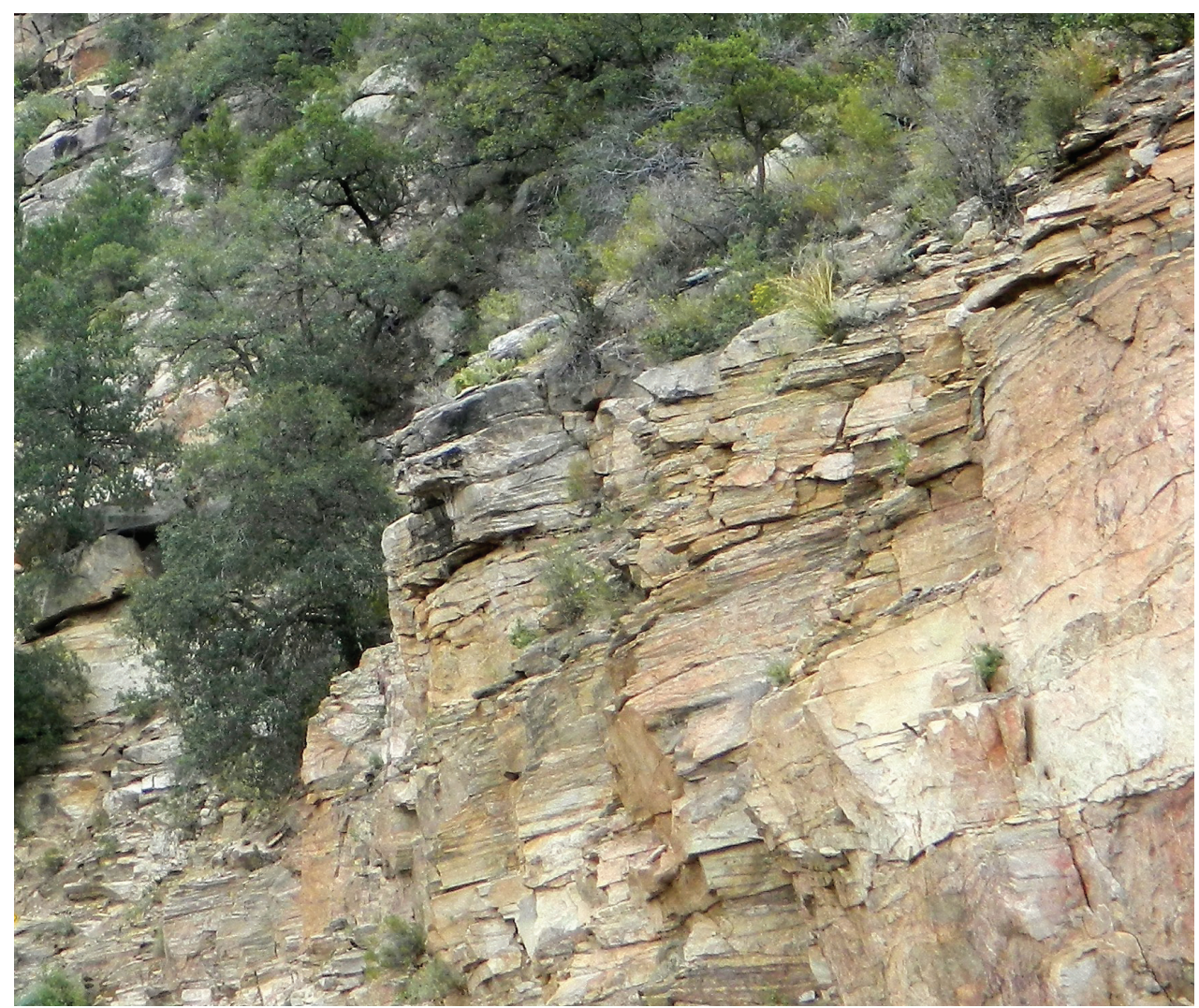

Figure I I. Vaejovis brysoni sp. n. habitat.

total length 10.44; femur length/width 2.69/1.00; patella length/width 2.75/1.06; chela length 5.00; palm length/width/depth 2.44/1.25/1.13; movable finger length 2.69; fixed finger length 2.56. Male paratype: total length 21.1; carapace length 2.75; mesosoma length 5.25; metasoma length 13.1; Metasoma: segment I length/ width/depth 1.44/1.81/1.25; segment II length/width/depth 1.56/1.88/1.13; segment III length/width/depth 1.75/1.63/1.19; segment IV length/width/depth 2.38/1.63/1.19; segment $\mathrm{V}$ length/width/depth 3.25/2.26/1.13. Telson: length 2.69; vesicle length/width/depth 1.81/1.00/1.00; aculeus length 0.88 . Pedipalps: total length 9.5; femur length/width 2.44/0.81; patella length/width 2.63/0.88; chela length 4.44; palm length/width/depth 1.88/1.06/1.06; movable finger length 2.69; fixed finger length 2.56 .

Distribution and natural history. Vaejovis brysoni sp. n. is known only from the type locality above Molino Basin on the Catalina Highway near the Seven Cataracts Vista, Santa Catalina Mountains, Arizona, USA. The type localities of the 12 described species in the vorbiesi group from Arizona and western New Mexico are shown in Figure 13. Vaejovis brysoni sp. n. is widely allopatric with $V$. halli, V. vorhiesi, V. cashi, V. feti, and V. electrum (Fig. 13). Vaejovis brysoni sp. n. and V. deboerae both occur within 

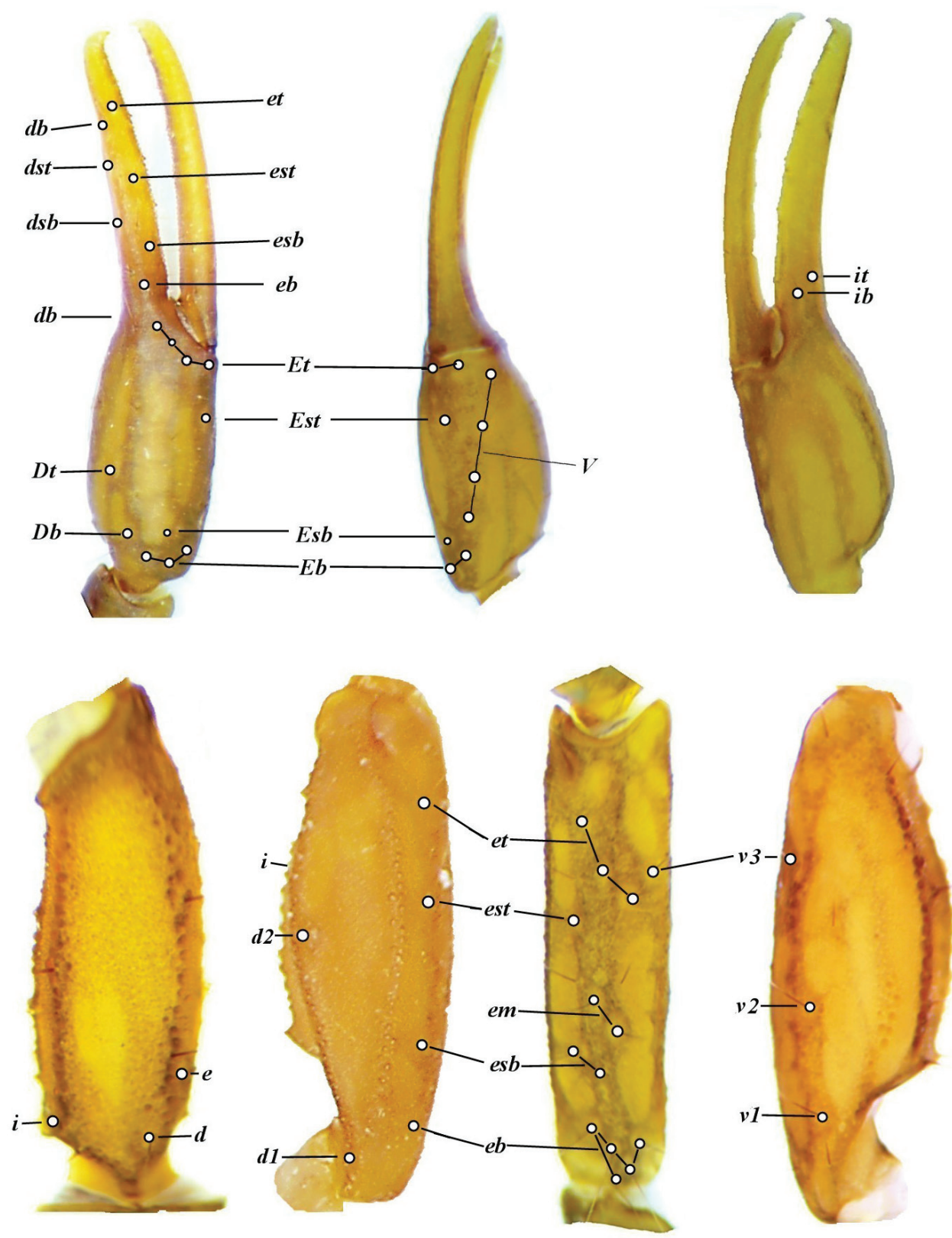

Figure 12. Vaejovis brysoni sp. n. trichobothrial pattern.

the Santa Catalina Mountains, and their ranges may overlap, perhaps along the midelevation pine-oak woodlands between 1800-1900 m.

The type specimens were found at night using a UV flashlight alongside the Catalina Highway. This area lies within open oak woodland and the transition zone from drier desert grassland to pine-oak woodland (Whittaker and Niering 1965). 


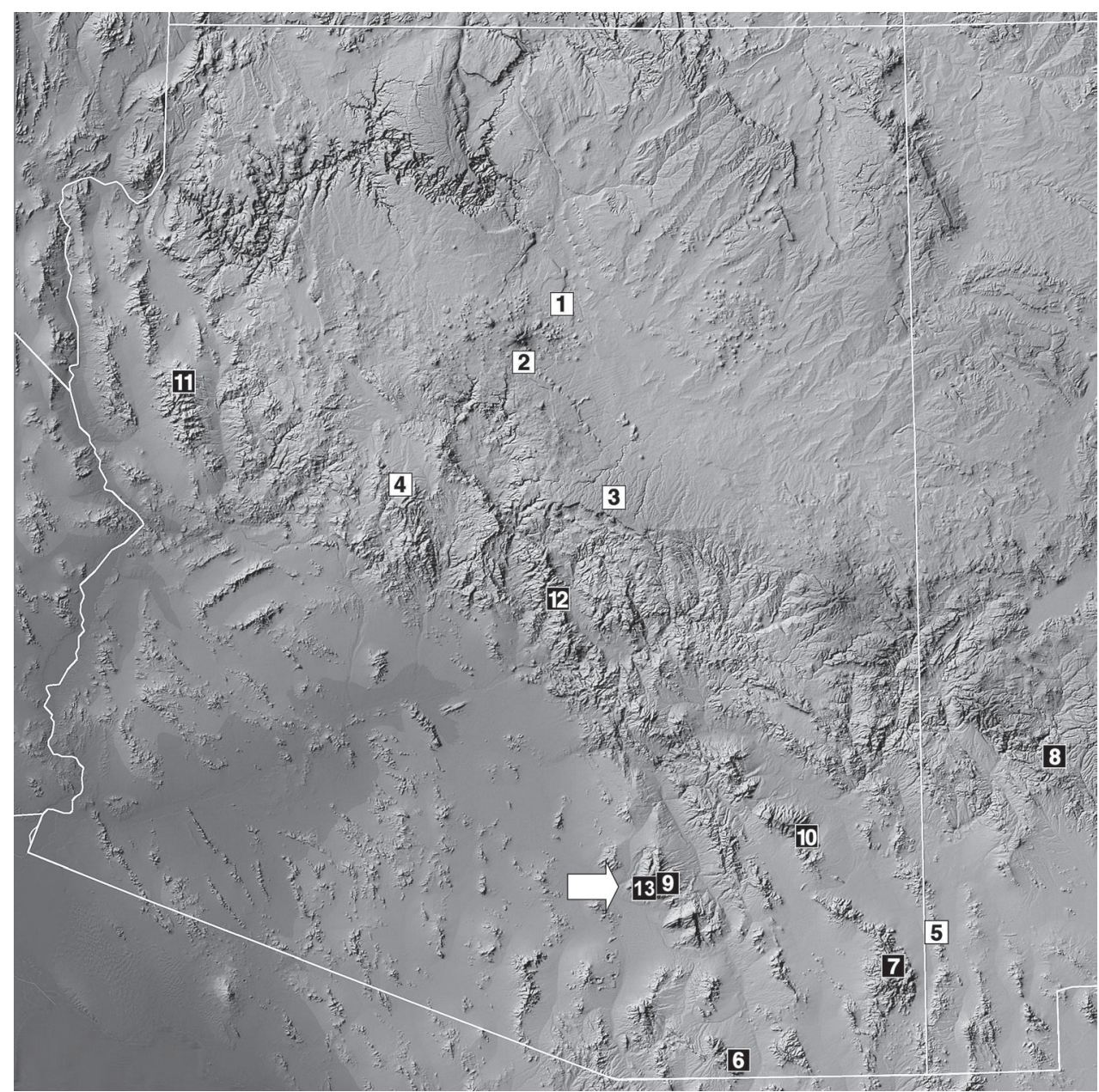

Figure 13. Map of Arizona and extreme western New Mexico showing the type localities of 13 of the 14 species in the Vaejovis vorhiesi group discussed in this paper, including the new species Vaejovis brysoni sp. n. The $V$. bandido type locality is south of Arizona in Sonora, Mexico. Localities are divided into those species exhibiting seven inner denticles $(I D)$ on the chelal movable finger (white rectangles with black numbering) and those with primarily six $I D$ denticles (black rectangles with white numbering). Seven IDs: I V. jonesi $\mathbf{2}$ V. lapidicola $3 \mathrm{~V}$. paysonensis $\mathbf{4} \mathrm{V}$. crumpi and $\mathbf{5} \mathrm{V}$. bigelowi. Six IDs: $\mathbf{6} \mathrm{V}$. vorhiesi $\mathbf{7} \mathrm{V}$. cashi $8 \mathrm{~V}$. feti $\mathbf{9} \mathrm{V}$. deboerae $\mathbf{I} \mathbf{0} \mathrm{V}$. electrum II V. tenuipalpus $\mathbf{I} \mathbf{2}$ V. halli and $\mathbf{I} \mathbf{3}$ V. brysoni sp. n.

Several Pseudouroctonus apacheanus (Gertsch \& Soleglad, 1972) and Centruroides sculpturatus Ewing, 1928 were also observed. In August of 2012, three captive female $V$. brysoni were observed with first instar juveniles (Fig. 3). The mean juvenile count was 23.67. The $1^{\text {st }}$ instar orientation on the mother's back was non-random, as is seen with many other species of Vaejovis (Hjelle 1974). They were facing anteriorly with the prosoma down and the metasoma raised over the prosoma of the juvenile immediately posterior to them. 


\section{Acknowledgments}

We would like to thank Dr. Robert W. Bryson Jr. for originally discovering the scorpions, Dr. Matthew R. Graham for valuable comments on earlier versions of this manuscript and two anonymous reviewers.

\section{References}

Ayrey RF (2009) Sky island Vaejovis: A new species (Scorpiones: Vaejovidae). Euscorpius 86: $1-2$.

Ayrey RF (2012) A new Vaejovis from the Mogollon Highlands of northern Arizona (Scorpiones: Vaejovidae). Euscorpius 148: 1-13.

Ayrey RF, Soleglad, ME (2011) A new species of Vaejovis from Prescott, Arizona (Scorpiones: Vaejovidae). Euscorpius 114: 1-15.

Fet V, Soleglad ME (2005) Contributions to scorpion systematics. I. On recent changes in high-level taxonomy. Euscorpius 31: 1-13.

Fet V, Soleglad ME (2007) Synonymy of Parabroteas montezuma Penther, 1913 and designation of neotype for Vaejovis mexicanus C. L. Koch, 1836 (Scorpiones: Vaejovidae). Boletín de la Sociedad Entomológica Aragonesa 41: 251-263.

Graham MR (2007) Sky island Vaejovis: two new species and a redescription of $V$. vorhiesi Stahnke (Scorpiones: Vaejovidae). Euscorpius 51: 1-14.

Graham MR, Bryson RB (2010) Vaejovis montanus (Scorpiones: Vaejovidae), a new species from the Sierra Madre Occidental of Mexico. Journal of Arachnology 38: 285-293. doi: 10.1636/Ha09-90.1

Graham MR, Ayrey RF, Bryson RB (2012) Multivariate methods support the distinction of a new highland Vaejovis (Scorpiones: Vaejovidae) from the Sierra de los Ajos, Mexico. Journal of Arachnology 40: 281-290. doi: 10.1636/Ha11-78.1

Hjelle JT (1974) Observations on the birth and post-birth behavior of Syntropis macrura Kraepelin (Scorpiones: Vaejovidae). Journal of Arachnology 1: 221-227.

Hughes GB (2011) Morphological analysis of montane scorpions of the genus Vaejovis (Scorpiones: Vaejovidae) in Arizona, with revised diagnoses and description of a new species. The Journal of Arachnology 39: 420-438. doi: 10.1636/Ha11-07.1

Sissom WD, Hughes GB, Bryson Jr RW, Prendini L (2012) The vorhiesi group of Vaejovis C. L. Koch, 1836 (Scorpiones: Vaejovidae), in Arizona, with description of a new species from the Hualapai Mountains. American Museum Novitates 3742: 1-19. doi: 10.1206/3742.2

Soleglad ME, Fet V (2006) Contributions to scorpion systematics. II. Stahnkeini, a new tribe in scorpion family Vaejovidae (Scorpiones: Chactoidea). Euscorpius 40: 1-32.

Soleglad ME, Fet V (2008) Contributions to scorpion systematics. III. Subfamilies Smeringurinae and Syntropinae (Scorpiones: Vaejovidae). Euscorpius 71: 1-115.

Soleglad ME (1973) Scorpions of the Mexicanus group of the genus Vaejovis. Wasmann Journal of Biology 31: 351-372. 
Soleglad ME, Lowe G, Fet V (2007) Systematic observations on the scorpion genus Syntropis, with description of two new species (Scorpiones: Vaejovidae). Boletín de la Sociedad Entomológica Aragonesa 40: 119-136.

Soleglad ME, Sissom WD (2001) Phylogeny of the family Euscorpiidae Laurie, 1896: a major revision. In: Fet V, Selden PA (Eds) Scorpions 2001. In memoriam Gary A. Polis. Burnham Beeches, Bucks: British Arachnological Society, 25-111.

Stahnke HL (1940) The scorpions of Arizona. Iowa State College Journal of Science 15: 101-103.

Stahnke HL (1970) Scorpion nomenclature and mensuration. Entomological News 81: 297-316.

Vachon M (1974) Etude des caractères utilizés pour classer les familles et les genres de Scorpions (Arachnides). 1. La trichobothriotaxie en Arachnologie. Sigles trichobothriaux et types de trichobothriotaxie chez les Scorpions. Bulletin du Museum National d'Historie Naturelle 140: 857-958.

Whittaker RH, Niering WA (1965) Vegetation of the Santa Catalina Mountains, Arizona: A gradient analysis of the south slope. Ecology 46: 429-452. doi: 10.2307/1934875 